\title{
Les défis bioéthiques - Vue de France
}

\section{Jean Martin}

Dr méd., membre de la rédaction

\section{Roger Gil}

Les grandes questions de bioéthique

$\mathrm{Au} \mathrm{XXI}{ }^{\mathrm{e}}$ siècle et dans le débat public

Bordeaux: LEH Editions; 2018

465 pages. 31 EUR.

ISBN 978-2-848747675

Roger Gil est neuropsychiatre et a été doyen de la Faculté de médecine de Poitiers. Il est engagé de longue date dans le domaine de la bioéthique, dont il a introduit l'enseignement, y compris en sciences politiques. Il publie un ouvrage très fourni montrant sa grande connaissance du champ, couvrant en 16 chapitres l'ensemble de la bioéthique et des débats y relatifs, en se référant notamment aux valeurs françaises dites républicaines.

Les quatre premiers chapitres plantent le décor des valeurs en cause et des droits de l'homme. A côté de thèmes qu'on peut dire classiques - enjeux éthiques des greffes d'organes, de la vie commençante (y compris procréation assistée) et finissante -, il se penche aussi sur les coûts de santé et leur régulation, la médecine prédictive, les soins sous contrainte, le pacte d'Ulysse à propos de directives anticipées en psychiatrie (Ulysse qui ordonne à ses compagnons, dont il y a bouché les oreilles, de l'attacher au mât et de ne lui obéir en aucun cas quand il entendra le chant des sirènes). Il discute, en rapport avec la question très française de la laïcité, la place de la chose religieuse. Un chapitre est dédié à la réglementation de la recherche scientifique, un autre à «Doit-on mériter ses soins?» (la réponse est non dans nos pays, sauf exception, mais il en va différemment aux USA par exemple); un autre encore au transhumanisme et un à une éthique globale de l'homme avec/ dans son environnement.

\section{Pluralisme des positions, une chance}

L'auteur se préoccupe de ne pas rester dans des ornières dogmatiques. Tout en présentant généralement les positions majoritaires françaises, il accorde un intérêt réitéré aux options théoriques et pratiques dans d'autres pays et contextes socio-culturels. "Alors que les démocraties pluralistes s'accordent sur les valeurs qui fondent les droits de l'Homme et qui infusent le monde de la santé, des divergences foisonnent qui surgissent de l'interprétation de ces valeurs comme des actions concrètes qui s'ensuivent.» "Cela peut donner l'impression d'un relativisme [...] Or, la pluralité doit être conçue comme une chance, du point de vue de la nécessaire tolérance et pour apprendre les exigences de la délibération morale.» De telles divergences existant entre pays proches par ailleurs, la démarche de Gil est plus fructueuse que, par hypothèse, s'en tenir à des affirmations réitérées sur le caractère intangible, jamais négociable, de principes qui seraient indissociables du statut de société civilisée... Plus adéquat de reconnaître que nous différons, sans doute, mais que nous pouvons apprendre l'un de l'autre.

\section{Respect de la vie, éthique}

Si la vie humaine est une valeur républicaine, son respect comporte des exceptions dont certaines sont universelles et d'autres variables d'un pays à l'autre: ainsi pour peine de mort, statut de l'interruption de grossesse, soins et démarches en fin de vie. Sur ce dernier sujet: «Les réponses de la République ont été les soins palliatifs, la règle du double effet, le droit à la sédation profonde jusqu'à la mort. D’autres pays ont choisi la voie du suicide assisté et de l'euthanasie. Mais le respect est rassemblé dans les relations interhumaines dans deux principes. Le premier, minimal et universel, est celui de non-malfaisance; le second, optionnel, est celui de bienfaisance.»

Remarque générale: "Le monde de la santé doit, à l'image de la société, éviter l'écueil d'une éthique maximaliste, où la bienfaisance dériverait vers le paternalisme, et celui d'une éthique minimaliste qui se satisferait de la seule non-nuisance à autrui.»

\section{Soins palliatifs et/vs aide à mourir}

Gil insiste à juste titre sur l'importance des soins palliatifs, tout en craignant que l'introduction éventuelle d'assistances à mourir soit au détriment de leur développement. «La légalisation d'une aide active à mourir introduirait une culture euthanasique à contre-cou- 
rant de la culture palliative. Car il ne s'agira pas de limiter l'euthanasie aux échecs des soins palliatifs mais d'instituer, au nom de l'autonomie, un nouveau droit.» C'est là un postulat de sa part, regrettable, avec lequel il convient de diverger au vu des expériences faites ailleurs. En effet, même si les palliatologues ne sont pas unanimes et si certains craignent vivement des "pentes glissantes» vers l'euthanasie, la tendance s'affirme qui montre que soins palliatifs et aide à mourir ne sont pas antagonistes - voir notamment les travaux de G.D. Borasio, de Lausanne [1]. En France, Véronique Fournier montre que médecine palliative et, par exemple, la sédation terminale maintenant admise peuvent être complémentaires, devenant des «soins palliatifs intégraux» [2].

\section{Procréation médicalement assistée}

Gil parle de "confusion française», évoquant par exemple la paternité post-mortem. Ainsi qu'à propos de pénurie d'ovocytes - alors que le don est légal, ce qui fait que la large majorité des couples français recourant à un tel don le font à l'étranger. Il le met en rapport avec des dispositions réglementaires et l'exigence ici aussi de la gratuité (alors qu'il paraîtrait normal que les contraintes pour la donneuse donnent lieu à compensation). Il discute la question de plus en plus aiguë de l'accès à ses origines, à propos duquel la France est en difficulté éthique et juridique croissante au plan international - avec le maintien à ce stade de l'accouchement sous $\mathrm{X}$ et de l'anonymat des donneurs. Gil: «En matière de bioéthique, la France n'en est pas à un paradoxe près [...] la question posée à la conscience de chacun est de savoir si l'accès aux origines doit ou non être considéré comme un droit fondamental et s'il est acceptable que la République détienne des renseignements sur des citoyens et refuse de les leur communiquer.» A noter aussi la présentation de la saga des débats récents autour de la GPA (grossesse pour autrui) en France.

\section{Spiritualité et laïcité}

A propos du spirituel dans la santé, que considère l'OMS aussi: «La spiritualité ne menace pas la laïcité puisqu'elle renvoie à la liberté de pensée dont se porte garant l'Etat laïc. La dimension spirituelle est constitutive de l'humain et l'attention à la spiritualité est une nécessité éthique. Encore ne faut-il pas se méprendre sur les missions des professions de la santé et pointer le danger d'un amateurisme relationnel. Le soignant n'est pas un directeur spirituel. Sa mission est de permettre aux besoins spirituels de s'exprimer et à la personne malade, si elle le souhaite, d'accéder à un aumônier.»

\section{Quelques autres aspects}

Rappel d'une problématique d'importance: "Il reste aussi à évaluer les conséquences de choix personnels sur la santé publique. La vaccination en est l'exemple typique» - en ne vaccinant pas ses enfants, on met en danger leur vie, mais aussi celle d'autres.

Transhumanisme: Nous arrive l'immortalité numérique, sera-t-elle suivie de l'immortalité biologique? Aussi: «La question fondamentale est de savoir si un groupe d'humains peut imposer à sa descendance son propre projet, non seulement en définissant son génome conformé aux désirs des parents, mais en remettant en question les conditions même d'une vie sur une planète partagée.»

A noter ce que Gil écrit sous «La vie humaine, vie environnée d'autres vies»: «L'être humain se sent investi de droits à l'égard de son environnement: il pêche et chasse, élève des animaux pour les consommer, il continue de déforester [...] Le respect de la vie suscité par les conséquences délétères d'actions humaines procède d'abord de mobiles rationnels, la prise de conscience de notre interdépendance avec le milieu. Mais s'y ajoutent l'émotion et l'admiration pour les formes de vie qui nous environnent.»

\section{En conclusion}

«L'attachement aux valeurs de la République nécessite plus que jamais qu'elles soient argumentées et confrontées à un nouveau mode du vivre ensemble.» [...] «Quand les valeurs entrent en conflit, des arbitrages divers induisent des lois différentes d'un pays à l'autre. Au sein d'un même pays, certaines lois rassemblent et d'autres opposent. Mais dans les lois se construit une anthropologie. La pluralité impose de faire le constat des fractures, afin que la diversité devienne un facteur de conscientisation.»

Dernières lignes: «La méditation sur les valeurs impose à chacun de se sentir comptable d'une humanité angoissée par sa finitude, tourmentée par le mal commis et par le mal subi et qui, dans le clair-obscur des parcours de vie, doit tenter de ne pas céder aux mirages.»

Les grandes questions de bioéthique est un ouvrage très complet, richement informé et argumenté, équilibré, de lecture aisée.

\section{Références}

1 Martin J. L'autonomie en fin de vie (à propos de l'ouvrage de ce titre de G.D. Borasio). Bull méd suisses. 2018;99(05):166-7.

2 Martin J. Le long cheminement en France de la réflexion sur la fin de vie (à propos de «La mort est-elle un droit?», de Véronique Fournier). Bull méd suisses. 2017;98(09):293. 